\title{
A novel numerical approach for the modelling of the square shaped silicon membrane
}

\author{
Fouad Kerrour and Farida Hobar \\ Laboratory of Microsystems and Instrumentation, University Mentouri - Constantine \\ Route d' Ain El Bey, 25000 Constantine, Algérie \\ Phone: 0709320 92, fax: 00213318190 10, \\ E-mail: fouadkerrour@caramail.com; f.hobar@caramail.com
}

\begin{abstract}
In this paper, we are interested in solving the Lagrange partial differential equation of the fourth order, which governs the deformation of a thin silicon square membrane, perfectly embedded and subjected to a uniform static pressure in the case of the weak disturbances. The proposed approach that consists of using the Galerkin method with trigonometrical basis functions is simple, easy to implement and ensures a good stability of the algorithm and a satisfactory accuracy.
\end{abstract}

Keywords: constraints, deformation, membrane, the Galerkin method.

Manuscript received 11.04.06; accepted for publication 23.10.06.

\section{Introduction}

The study of the deformation of a membrane perfectly embedded at the edges is very significant in the realization of the pressure microsensors. Indeed, the knowledge of the membrane response to an external pressure excitation makes it possible to determine its characteristics and performances. The exact solution of this type of the problem does not exist. However, several approaches were proposed, in particular, the Ritz Raleigh method and the Galerkin method [1-7].

Ben Moussa [6] and Naciri [7] proposed a solution using the Galerkin method with a polynomial approximation. The study of the results obtained highlighted the limits of this model in accuracy as well as in convergence. Theoretically, this method is more accurate when the number of the basis functions becomes high. However, for the models described by Ben Moussa [6] and Naciri [7], the precision condition is not verified, because the algorithm is numerically unstable.

Accordingly, we propose to improve this technique by using trigonometrical basis functions. The analyzed structure is a membrane of a square shape. This membrane is supposed to be perfectly embedded at the edges and subjected to a constant static pressure as shown in Fig. 1. The problem is based on the principle of the minimization of energy under the assumption of weak disturbances.

The paper is organized as follows. In the first section, we recall the establishment of the partial differential equation that governs the deflection of a membrane perfectly embedded at the edges and subjected to a constant static pressure. The second section is devoted to the mathematical formulation of the equations allowing the analytical semi solution of the Galerkin method. We present then the numerical solution of the linear problem $A \vec{k}=\vec{b}$. Section 4 will be devoted to the presentation of the results obtained and a comparative study between these two models used. In conclusion, we comment the main results obtained by specifying the impact of this study on the realization of the pressure microsensors.

\section{Establishment of the fundamental equation of the membrane deflection}

\subsection{Geometry of the membrane}

The membrane of rectangular or square shape of silicon is described in Fig. 1. It is a microstructure directed according to the crystallographic plane (110) deposited on the substrate of orientation (100) [8], dimensions of which are: the length $2 a$ according to the OX axis, width $2 b$ according to the OY one, height $h$ according to the OZ one with $h<<2 a$ and $h<<2$ b (assumption of thin section). We define $R$ as the ratio of membrane dimensions such as $R=b / a$. In our study, we consider the case, where the membrane is square, then we take $R=1$ and $a=b$.

According to the theory of the thin sections [9], the mechanical behaviour of a membrane subjected to a uniform and static pressure $P$ in the case of the weak disturbances, $w<<h$, is governed by the following Lagrange equation of the fourth order: 


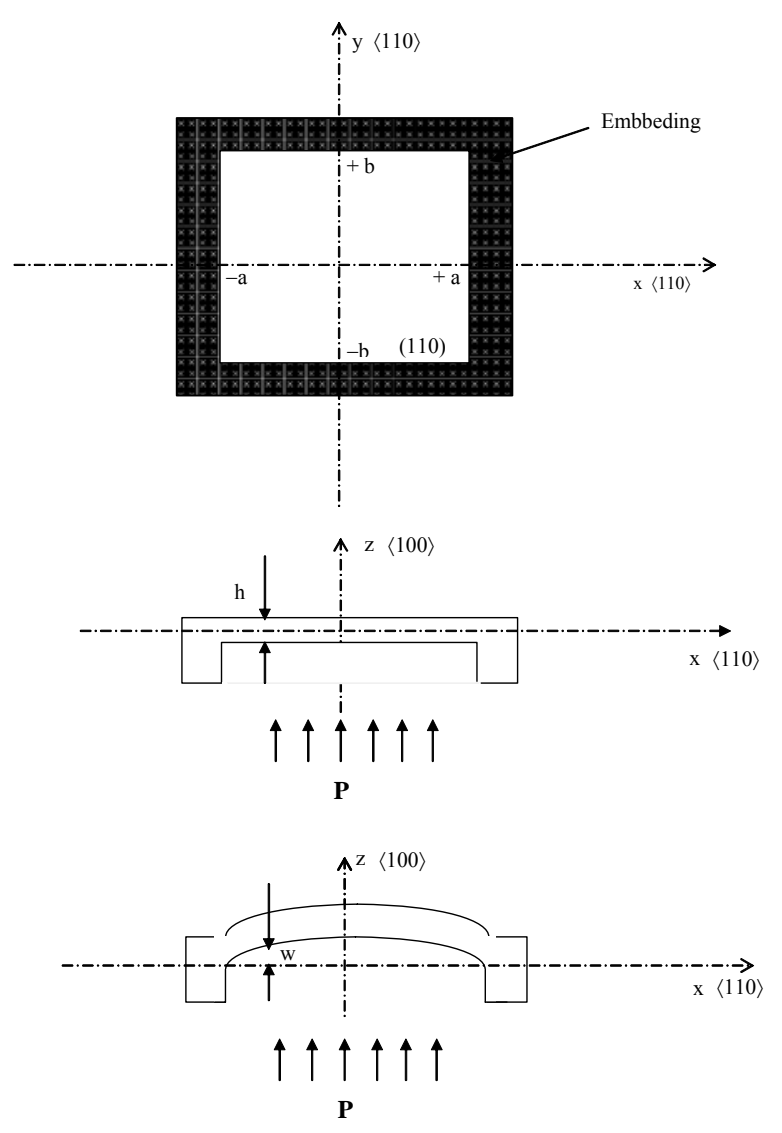

Fig. 1. Schematic top-view of the structure (a); $a$-sections of the structure over the xoz plane with $P=0$ (b) and with applied $P(\mathrm{c})$.

$\frac{\partial^{4} w(x, y)}{\partial x^{4}}+2 \alpha_{\mathrm{Si}} \frac{\partial^{4} w(x, y)}{\partial x^{2} \partial y^{2}}+\frac{\partial^{4} w(x, y)}{\partial y^{4}}=\frac{P}{D}$

with $\alpha_{\mathrm{Si}}$ is the coefficient characterizing the anisotropy of the silicon material, which is defined by [9-10]

$\alpha_{\mathrm{Si}}=v+\frac{2 G}{E}\left(1-v^{2}\right)$

and $D$ is the coefficient of rigidity of the material, which is given by

$D=\frac{E h^{3}}{12\left(1-v^{2}\right)}$,

where $v$ is the Poisson ratio, $E$ is the Young modulus and $G$ is the Coulomb (shear) modulus. The boundary conditions imposed by the embedding of the membrane on its board $[10,11]$ are:

$$
\begin{aligned}
& w(x= \pm a, \forall y)=0, \\
& w(\forall x, y= \pm b)=0, \\
& \frac{\partial w}{\partial x}(x= \pm a, \forall y)=0,
\end{aligned}
$$

$\frac{\partial w}{\partial y}(\forall x, y= \pm b)=0$

\subsection{The method of Galerkin}

This method is based on the variational formulation that is the starting point for the solution by using the method of finite elements and the spectral methods. It consists of choosing orthogonal basis functions $\phi_{i j}(x, y)$ such as:

$$
\left\langle\phi_{i j}(x, y) \otimes \phi_{i j}(x, y)\right\rangle=\delta_{i k} \times \delta_{j m} .
$$

The setting equation of the Galerkin method consists of finding the variational form of the equilibrium equation (1) corresponding to the problem which is obtained starting from the minimization of the functional calculus $F\left(\phi_{i j}(x, y)\right)[12,13]$

$$
\begin{aligned}
& F\left(\phi_{i j}(x, y)\right)=\oiint_{D}\left[\frac{\partial^{4} \phi_{i j}(x, y)}{\partial x^{4}}+\right. \\
& \left.+2 \alpha_{\mathrm{Si}} \frac{\partial^{4} \phi_{i j}(x, y)}{\partial x^{2} \partial y^{2}}+\frac{\partial^{4} \phi_{i j}(x, y)}{\partial y^{4}}-\frac{P}{D}\right] \phi_{i j}(x, y) \partial x \partial y=0
\end{aligned}
$$

where $\phi_{i j}(x, y)$ is the basis function that must be orthogonal and verify the boundary conditions of the problem governed by (1). $D$ is the field of integration along the membrane surface. From (6), we find $a_{i j}$ and $b_{i j}$ that allows us to solve the linear problem $A \vec{k}=\vec{b}$ numerically, where $a_{i j}$ and $b_{i j}$ are, respectively, the elements of the matrices [A] and [B] described by the following equations:

$a_{i j}=\oiint\left[\frac{\partial^{4} \phi_{i j}(x, y)}{\partial x^{4}}+2 \alpha_{\mathrm{Si}} \frac{\partial^{4} \phi_{i j}(x, y)}{\partial x^{2} \partial y^{2}}+\right.$

$\left.+\frac{\partial^{4} \phi_{i j}(x, y)}{\partial y^{4}}\right] \phi_{i j}(x, y) \partial x \partial y$,

$b_{i j}=\oiint \frac{P}{D} \phi_{i j}(x, y) \partial x \partial y=0$

and the coefficients $k_{i j}$ of the matrix [K] are the unknown factors to be determined numerically.

We can then substitute the deformation expression $w(x, y)$ by the basis functions expression $\phi_{i j}(x, y)$ in the equation (1) and, thus, we obtain the following equation:

$$
\begin{aligned}
& \sum_{i=0}^{n} \sum_{j=0}^{n} k_{i j}\left[\frac{\partial^{4} \phi_{i j}(x, y)}{\partial \mathrm{x}^{4}}+\right. \\
& \left.+2 \alpha_{\mathrm{Si}} \frac{\partial^{4} \phi_{i j}(x, y)}{\partial x^{2} \partial y^{2}}+\frac{\partial^{4} \phi_{i j}(x, y)}{\partial y^{4}}\right]=\frac{P}{D}
\end{aligned}
$$

by carrying out all the analytical calculations, from the equations (7), (8) we obtain the elements $a_{i j}, b_{i j}$ of the 
matrices [A] and [B] that define the system of linear equations where the elements $k_{i j}$ are to be determined

$$
[A][K]=[B] \text {. }
$$

After numerical solution of this equation, we obtain the expression of the deflection $w(x, y)$ at any point of the membrane:

$$
w(x, y)=\sum_{i=0}^{n} \sum_{j=0}^{n} k_{i j} \phi_{i j}(x, y) \text {. }
$$

\section{Choice of the basis functions: polynomial model}

The choice of the type and the number of the basis functions to be used is conditioned by a better approximation of the real solution (convergence to the solution) and of the execution time of calculations. For the sake of comparison, we first carry out the work done in $[6,7]$ which uses the two-dimensional polynomial function of the type:

$\phi_{i j}(x, y)=\left(1-\left(\frac{x}{a}\right)^{2}\right)^{2}\left(1-\left(\frac{y}{b}\right)^{2}\right)^{2}\left(\frac{x}{a}\right)^{i}\left(\frac{y}{b}\right)^{\mathrm{j}}$

with $i, j$ even numbers. This basis function satisfies the boundary conditions imposed by the perfect embedding of the membrane (4a)-(4d). We will substitute this expression of the basis function into equation (9), which makes it possible to obtain the solution of the linear equations system (10).

\section{Numerical solution}

The numerical solution of the system of linear equations (10), using Mat lab, makes it possible to determine the standardized coefficients $k_{i j}, k_{i j}=k_{i j} / k_{00}$ that are summerized in Table 1.

We note that these results are identical to those obtained in $[6,7]$ for $n=3$. As the Galerkin method is more accurate when the number of basis functions is higher, we refined the model by using various values of $n(n=4,5,6,7, \ldots, 16$, etc.). Unfortunately, the results obtained show that:

- the coefficients $k_{i j}$ do not converge any more;

- the orthogonality condition of the matrix [A] is not valid any more;

- for $n=7$, the limiting value of this model, the matrix is singular and its numerical conditioning cannot be done.
This proves that this model is limited and not accurate.

From the previous observations, we were brought to consider new basis functions ensuring a better convergence to the solution, a reduced computing time as $n$ tends to infinity as well as a better accuracy.

\section{Proposed approach: trigonometrical model}

The choice of this basis function was made after having prospecting several types of functions; ultimately we choose the following function:

$$
\begin{aligned}
& \phi_{i j}(x, y)=\frac{1}{2}\left[\cos ^{2}\left(\frac{(2 i+1) \pi x}{2 a}\right)\right] \times \\
& \times \frac{1}{2}\left[\cos ^{2}\left(\frac{(2 j+1) \pi y}{2 b}\right)\right]
\end{aligned}
$$

where $i, j$ are the even numbers varying from 0 to $n$.

This trigonometrical basis function satisfies the Galerkin condition of equation (5) and the boundary conditions of equations (4a)-(4d).

Therefore, the deflection $w(x, y)$ could be calculated starting from expression (11). After doing all calculations, the solution of equation (10) makes it possible to determine the coefficients $k_{i j}$ that reconstitute the approximate expression of the deflection $w(x, y)$ at any point. The first conclusion one can make is that the basis functions used give a matrix [A] orthogonal which is the necessary Galerkin method condition. The results obtained for $n=3$ are summarized in Table 1 .

\section{Results and discussion}

From the given coefficients $k_{i j}$, we calculate the approximate value of the deformation of the membrane $w(x, y)$ at any point. This deformation is expressed as:

$$
\begin{aligned}
& w(x, y)=\left(1-\left(\frac{x}{a}\right)^{2}\right)^{2}\left(1-\left(\frac{y}{b}\right)^{2}\right)^{2} \times \\
& \times \sum_{i=0}^{n} \sum_{j=0}^{n} k_{i j}\left(\frac{x}{a}\right)^{i}\left(\frac{y}{b}\right)^{j},
\end{aligned}
$$

in the case of the polynomial model and

$$
\begin{aligned}
& w(x, y)=\frac{1}{4} \sum_{i=0}^{n} \sum_{j=0}^{n} k_{i j} \cos ^{2}\left(\frac{(2 i+1) \pi x}{2 a}\right) \times \\
& \times \cos ^{2}\left(\frac{(2 j+1) \pi y}{2 b}\right) .
\end{aligned}
$$

in the case of the trigonometrical model.

Table 1. Values of the reduced coefficients $\boldsymbol{k}_{\boldsymbol{i j}}$ for $\boldsymbol{n}=3$.

\begin{tabular}{|c|c|c|c|c|c|c|c|}
\hline Model & $k$ & $k_{00}$ & $k_{02}=k_{20}$ & $k_{22}$ & $k_{24}=k_{42}$ & $k_{40}=k_{04}$ & $k_{44}$ \\
\hline$[6]$ & 0.0220 & 1 & 0.2140 & 0.2700 & 0.0980 & -0.0062 & -0.1035 \\
\hline Polynomial & 0.0220 & 1 & 0.2143 & 0.2697 & 0.0985 & -0.0061 & -0.1057 \\
\hline $\begin{array}{c}\text { Trigono- } \\
\text { metrical }\end{array}$ & 0.0224 & 1 & 0.0284 & 0.0123 & 0.0030 & 0.0038 & 0.0016 \\
\hline
\end{tabular}


From the expressions (14) and (15), we plot the deflection curves $w(x, y)$ for $n=3$. For convenience, we use the standardized expression compared to the membrane dimensions. Thus, we define the maximum deflection expression in the center of the membrane [6] as

$w_{00}=k\left(P a^{2} b^{2}\right) / 16 D$,

where $k$ is the real constant and its value is given in Table 2 for different used models.

We represent the normalized deflection $\left[w(x, y) / w_{00}\right]$ and its contour lines in Fig. 2.

Substituting the expressions (14), (15) of the deflection $w(x, y)$ in the equations defining the constraints according to the deformation [9, 10], we obtain the normal and shearing components expressions of the tensor of the constraints $\sigma_{x x} \sigma_{y y} \sigma_{x y}$ as :

$$
\begin{aligned}
& \sigma_{x x}=-\frac{E z}{1-v^{2}}\left[\frac{\partial^{2} w(x, y)}{\partial x^{2}}+v \frac{\partial^{2} w(x, y)}{\partial y^{2}}\right], \\
& \sigma_{y y}=-\frac{E z}{1-v^{2}}\left[v \frac{\partial^{2} w(x, y)}{\partial x^{2}}+\frac{\partial^{2} w(x, y)}{\partial y^{2}}\right], \\
& \sigma_{x y}=-2 G z \frac{\partial^{2} w(x, y)}{\partial x \partial y} .
\end{aligned}
$$

From these expressions, we plot the curves of the $\sigma_{y y}(x, y)$ and $\sigma_{x y}(x, y)$ constraints for $n=3$, which are illustrated in Figs 3 and 4 . The curves of Figs $3 \mathrm{a}$ and $4 \mathrm{a}$ are identical to those found in $[6,7]$ that validates our calculations.

The comparison of the various curves obtained for these two models leads to some interesting points.

Our model emphasizes the existence of a critical deformation line at the median location in both directions, $x=0 \forall y$, and $\mathrm{y}=0 \forall x$, which bears a visible sharpness in the deformation profile, as it is shown in Fig. 2b. This critical line is the main feature revealed by the present model. It is a remarkable specifity of the real-world square shaped embedded membrane, which cannot be perceived through the results of the existing models from the literature up to date (compared with Fig. 2a).

As an immediate consequence, this critical line is the site of a significant peak in the magnitude of $\sigma_{y y}$ constraint (Fig. 3a), and a drastic discontinuity in the magnitude of $\sigma_{x y}$ constraint (Fig. 4b).

Table 2. Value of the constant $k$ for $n=3$, for $x=y=0$ for different models used.

\begin{tabular}{|c|c|c|c|}
\hline & {$[6]$} & Polynomial & Trigonometrical \\
\hline$k$ & 0.022000 & 0.022000 & 0.022415 \\
\hline
\end{tabular}
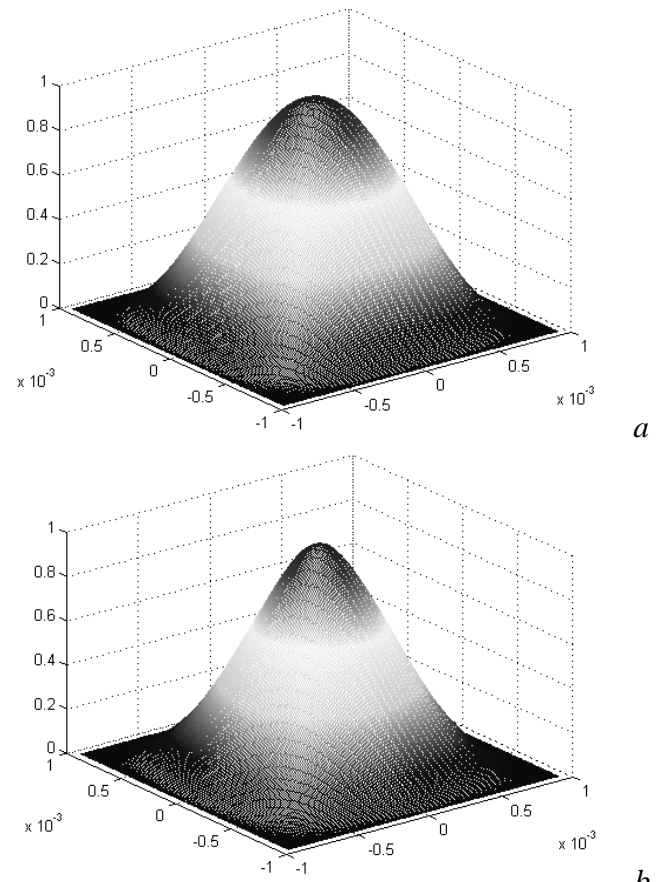

$b$
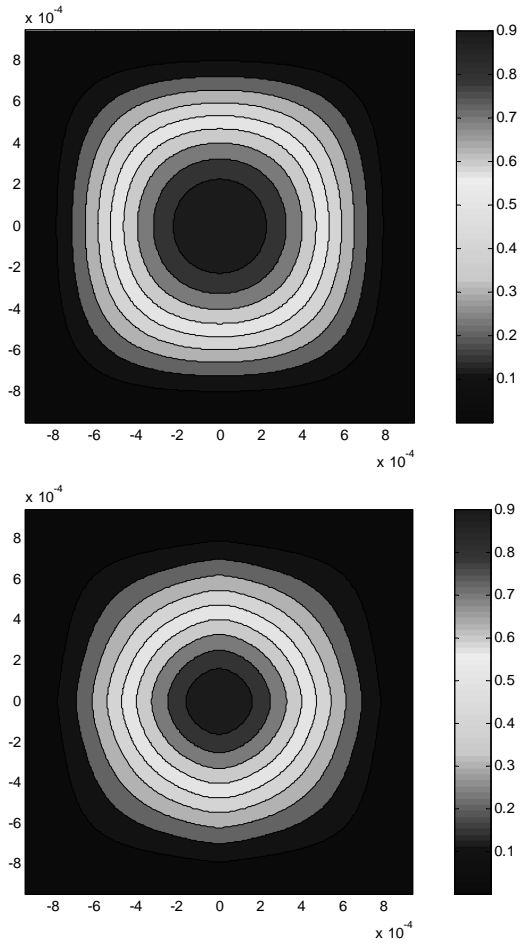

$d$

Fig. 2. a - polynomial model: normalized deflection $\left[w(x, y) / w_{00}\right] ; \mathbf{b}-$ trigonometrical model: normalized deflection $\left[w(x, y) / w_{00}\right] ; \quad c-$ polynomial model: normalized deflection $\left[w(x, y) / w_{00}\right]$ and its contour lines; $\mathrm{d}$ - trigonometrical model: normalized deflection $\left[w(x, y) / w_{00}\right]$ and its contour lines. 

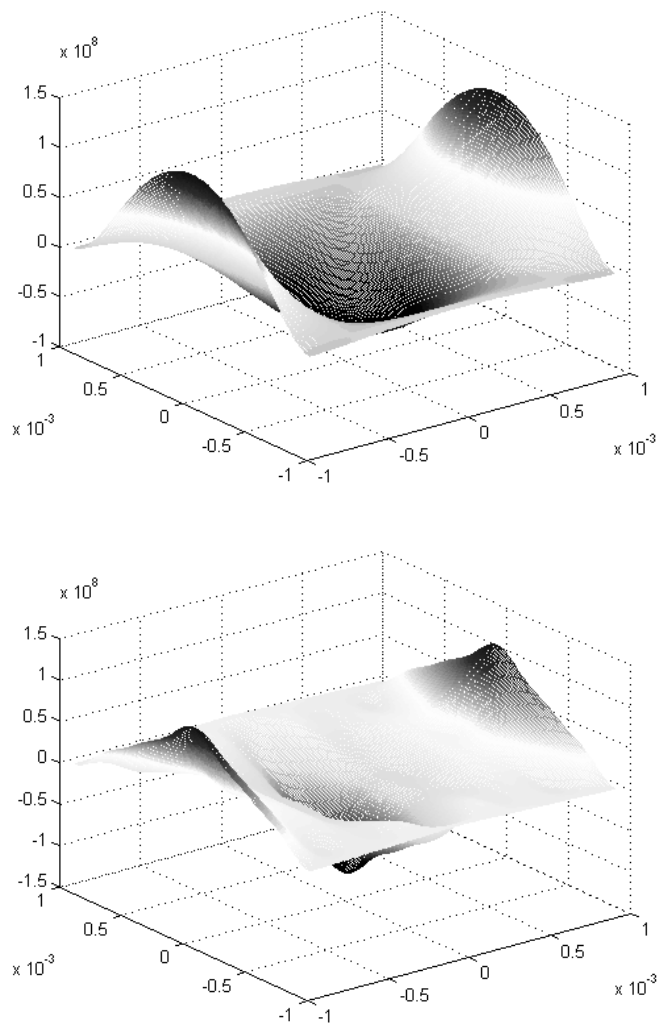

$b$

Fig. 3. a - polynomial model: normal stress $\sigma_{y y}(x, y)$; $\mathrm{b}$ - trigonometrical model: normal stress $\sigma_{y y}(x, y)$.

We predict that this remark would have an important technological impact in the design of optimized devices, in the sense that it makes possible an accurate knowledge of the optimal position of the strain gauges on the membrane. We have also plotted the curves of convergence of the coefficient $k_{i j}$ with respect to the number of basis functions used, $n$, for these two models for some values of $i$ and $j$. We note that our model converges more quickly. The curves are presented in Fig. 5. From the results of simulation we can affirm that:

1. For a number of basis functions $n=3$, the curves and the values of the standardized coefficients $k_{i j}$ obtained are those exactly obtained in [6,7]. These values were recalculated for the sake of comparison and also to confirm the exactness of our results.

2. The maximum deflection $w_{00}$ equals for polynomial case $w_{00}=4.6625 \cdot 10^{-6} \mathrm{UI}$, trigonometrical case $w_{00}=4.7529 \cdot 10^{-6} \mathrm{UI}$.

It is noted that the maximum value of the deflection is slightly higher in the trigonometrical case, that is to say an inaccuracy of about $2 \%$ tolerated by the polynomial model.

To estimate the error $\varepsilon$ of the approximation, we use the exact relation of the deflection $w(x, y)$ given by
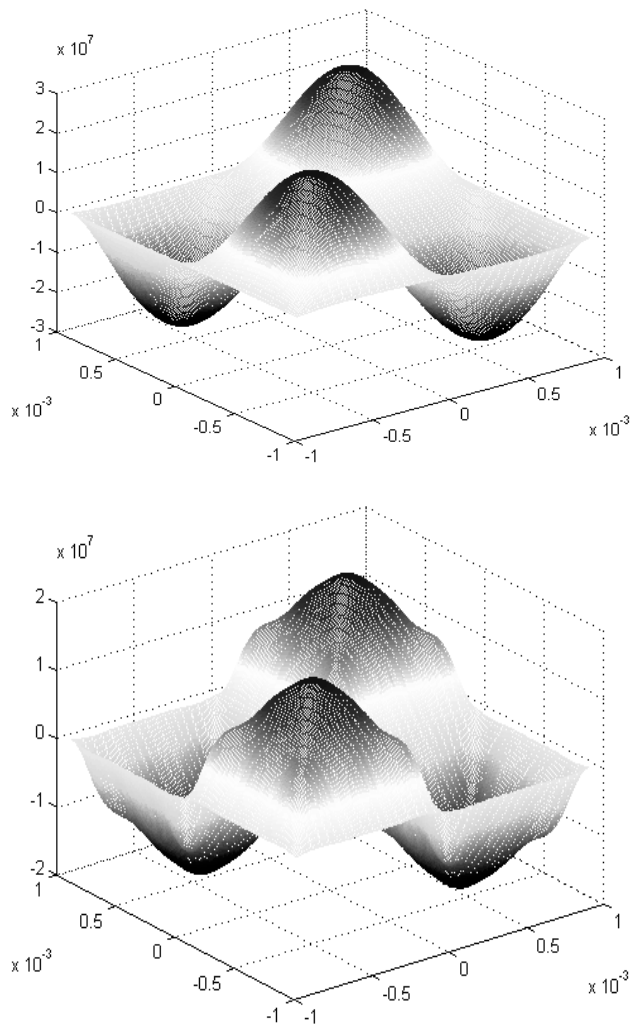

$a$

Fig. 4. a - polynomial model: shear stress $\sigma_{x y}(x, y)$; b - trigonometrical model: shear stress $\sigma_{x y}(x, y)$.
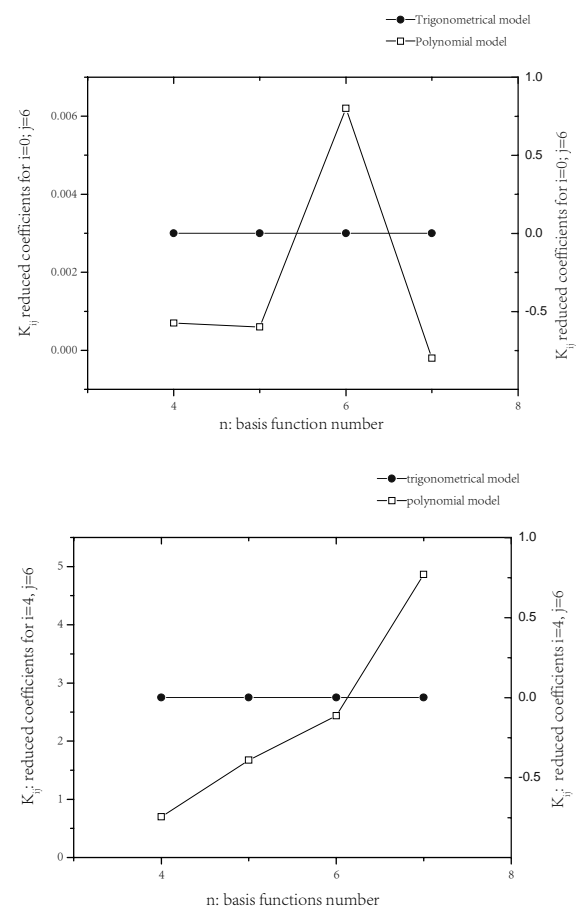

Fig. 5. Convergence curves $k_{i j}=f(n)$ for $i=0, j=6$ (a) and $i=4, j=6(\mathrm{~b})$. 
$w(x, y)=\sum_{i=0}^{n} \sum_{j=0}^{n} k_{i j} \phi_{i j}(x, y)+\sum_{i=n+1}^{\infty n} \sum_{j=n+1}^{\infty} k_{i j} \phi_{i j}(x, y)$,

where the error $\varepsilon$ is given by the expression

$\varepsilon=\sum_{i=n+1}^{\infty} \sum_{j=n+1}^{\infty} k_{i j} \phi_{i j}(x, y)$

From expression (18) and for $n=16$, the error $\varepsilon$ in our model is about $10^{-12}$. The values of the standardized coefficients $k_{i j}$ are smaller (about $10^{-14}$ ). For comparison, the error is about $10^{-3}$ in the polynomial model from $[6,7]$, and cannot be improved owing to the fact that the matrix [A] becomes singular if $n$ increases.

In the theory, the error $\varepsilon$ tends to zero as $n$ increases, however there exists a threshold $n$, beyond which the error does not vary any more in a significant way. This makes it possible to affirm that our model is more accurate, simpler to implement and with a much reduced computing time.

\section{Conclusions}

In this work, we highlighted the limits of the polynomial model, which can be summarized as follows:

1. The number of basis functions is limited to $n=3$, and increasing $n$ causes a deterioration of the convergence and stability of the model.

2. Relative increase in the computing time and a reduction in the precision. The absolute error does not tend to zero as $n$ tends to infinity.

The trigonometrical model allows to obtain more accurate results and with a reduced computing time.

Our model (that is confirmed by two objective mathematical criteria: the estimation of the relative error and the computation of convergence rate) is among all the approaches proposed in the literature the one which reproduces with the nearest technological conformity the real-world square shaped membrane.

\section{References}

1. D. Young, Vibration of rectangular plates by the Ritz method // J. Appl. Mechanic. 17, p. 448 (1950).

2. D. Maier-Schneider, J. Maibach, and E. Obermeier, A new analytical solution for the load-deflection of square membranes // J. Microelectromechanical Systems 4, p. 238-241 (1995).

3. C.S. Sander, J.W. Knutti and J.D. Meindel, A monolithic capacitive pressure sensor with pulse period output // IEEE Trans. on Electron Devices ED 27 (5), p. 927 (1980).

4. R. Steinmann, H. Friemann, C. Presher and R. Schellin, Mechanical behaviour of micro machined sensor membrane under uniform external pressure affected by in plane stresses using a Ritz method and Hermite polynomials // Sensors and Actuators A48 p. 37-46 (1995).

5. H.E. Elgamel, Closed form expressions for the relationships between stress, diaphragm deflection and resistance change with pressure in silicon piezoresistive pressure sensors // Sensors and Actuators A50, p. 17-22 (1995).

6. N. Ben Moussa, Conception, modélisation et réalisation d'un capteur de pression capacitif micro électronique. Thèse de doctorat d'état, LAAS, Toulouse, 1985.

7. Y. Naciri, Contribution à l'étude de capteur de pression capacitif miniaturisés. Thèse de doctorat d'état, LAAS, Toulouse, 1986.

8. L. Inden, L. Tenerz, J. Tiren and B. Hok, Fabrication of three dimensional silicon structure by means of doping selective etching // Sensors and Actuators 16, p. 67 (1989).

9. S.P. Timochenko, S. Woinoiwsky-Krieger, Theory of plates and shells. McGraw-hill, 1982.

10. S.P. Timochenko, J.N. Goodier, Theory of elasticity. Second edition, McGraw-hill, 1951.

11. J.J. Wortmans, R.A. Evans, Young's modulus, shear modulus and Poisson's ratio in silicon and germanium // J. Appl. Phys. 36 (1), p. 153 (1965).

12. Jacques Rappaz, Marco Picasso, Introduction à l'analyse numérique. Presses polytechniques et universitaires de Romandes, 2000, Ch-1015 Lausanne, Suisse.

13. C. Fletcher, Computational Galerkin method. Springer Verlag, 1984. 